\title{
TWO-STAGE MEMBRANE SEPARATION WITH CARRIER GAS FOR ETHANOL-WATER MIXTURES USING SILICONE RUBBER AND POLYIMIDE HOLLOW FIBERS
}

\author{
AKIRA ITO AND MICHIHIRo FUJII \\ Department of Chemical Engineering, Nïgata University, Niigata 950-21 \\ TAKASHI HARADA \\ Corporate Research \& Development, Ube Industries, Ltd., Chiba 290
}

Key Words: Membrane Separation, Silicone Rubber Membrane, Polyimide Membrane, Ethanol-Water Mixture, Pervaporation, Gas Membrane Separation

\begin{abstract}
A two-stage membrane separation process composed of one step using a silicone rubber membrane hollow-fiber module and another step using a polyimide hollow-fiber module for effective separation of alcohol from a low-concentration alcohol-water solution was proposed and its effectiveness was experimentally confirmed.

The first step is a pervaporation through the silicone rubber into a carrier gas, and the second is a gas-phase separation through the polyimide membrane. By use of these two steps in series, $99 \%$ and $1 \%$ solutions were separated from 5-10 mol\% ethanol solution.

This effective separation must be due to the high permeation rate of water vapor through the polyimide membrane, 200-fold that of ethanol vapor as shown by the experimental data.
\end{abstract}

\section{Introduction}

In recent years, membrane separation processes have been economically competing with conventional separation methods due to the advance of hollow-fiber technology. Because the number of the membrane materials suitable for hollow-fiber manufacturing on a commercial scale is limited, however, multiplemembrane separation systems that combine membranes with different characteristic properties to achieve efficient separation of specified mixtures have been tried.

Seok et $a l .^{12)}$ demonstrated the performance of a two-membrane column for pervaporation using cellulose acetate and silicone rubber hollow-fiber modules for the separation of water-ethanol and water-isopropanol azeotropic mixture. A two-membrane permeator composed fo these two kinds of hollow fibers was proposed by Seguputa et al. ${ }^{11)}$ for ternary gas separation processes.

Silicone rubber membranes can concentrate alcohol from dilute alcohol-water mixtures. Pervaporation of alcohol solutions using them has been reported by Hwang et al., ${ }^{1)}$ Kimura and Nomura ${ }^{3)}$ and Okamoto et al. ${ }^{8)}$ On the other hand, a polyimide hollow-fiber membrane has much larger permeability for water vapor than for ethanol vapor, as reported in the recent literature. ${ }^{5,7,8)}$

In this article, noting the difference in properties

\footnotetext{
* Received January 31, 1989. Correspondence concerning this article should be addressed to A. Ito.
}

between silicone rubber membranes and polyimide membranes, and expecting to achieve effective separation of each component from a solution of low alcohol concentration, we combined the two membrane modules in series in a separation system and carried out an experimental evaluation of its performance.

\section{Experimental}

\subsection{Membrane materials and columns}

Permeation columns were constructed of hollow fibers in a shell-and-tube configuration. A pervaporation module was made of silicone rubber hollow fibers (O. D./I. D.: $320 \mathrm{~mm} / 200 \mathrm{~mm}$ ) from Nagayanagi Kougyou Corp., Tokyo, Japan. A bundle of 3000 fibers was housed in a tube of $0.03 \mathrm{~m} \mathrm{I}$. D. The effective column length was $0.15 \mathrm{~m}$ and the permeation area was $0.32 \mathrm{~m}^{2}$. Another module for vapor-phase separation was made of polyimide hollow fibers (O. D. is smaller than $0.5 \mathrm{~mm}$ ) from Ube Industries Ltd., Tokyo, Japan. A bundle of 100 fibers was housed in a glass tube of $0.012 \mathrm{~m} \mathrm{I}$. D. The effective permeation area was $0.03 \mathrm{~m}^{2}$.

\subsection{Apparatus and procedure}

Figure 1 is a schematic diagram of the two-stage membrane separation process. The apparatus consists of a silicone rubber membrane coumn, a polyimide membrane column, a diaphragm vacuum pump, two sets of condensers and condensate collection tubes, and a recycle line for carrie gas, helium. The diaphragm vacuum pump, manufactured by KNF 


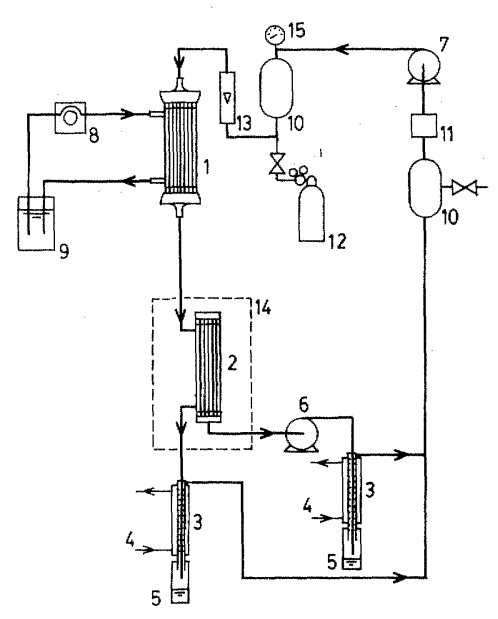

Fig. 1. Schematic diagram of two-stage membrane separation process
1: Silicone rubber column
9: Feed solution
2: Polyimide column
10: Buffer tank
3: Condenser
11: Mass flow controller
4: Coolant
12: Helium gas
5: Condensate collection tube
6: Vacuum pump
7: Circulation pump
3: Purge meter
14: Constant-temperature bath
15: Pressure gauge

\section{Neuberger}

$\mathrm{GmbH}$ in W. Germany, is connected to the tube-side outlet of the polyimide column. The condensers are of annular tube type with fins on the wall and have a wall area of $0.017 \mathrm{~m}^{2}$. All equipment units are connected by nylon tubing.

An ethanol-water liquid mixture of low concentration is pumped with a peristaltic pump to the shell side of the silicone rubber column. Pervaporation takes place through that membrane and enriched ethanol-water vapor permeates into the carrier gas. For the pervaporation column, both shell and tube sides are kept at atmospheric pressure and room temperature.

The ternary gas mixture composed of ethanol, water and helium flowing out of the pervaporation column is then introduced to the shell side of the polyimide column. Since the tube side of this column is maintained at low pressure by the vacuum pump, the vapor mixture permeates through the polyimide membranes at a finite stage-cut. This column is set in a conatant-temperature bath at $333.2 \mathrm{~K}$ to prevent vapors from condensing and to maintain a high permeation rate of mixed gas.

The reject and permeate streams flowing out of the polyimide column are cooled by the condensers, and the vapors contained in the carrier gas are collected as condensates. To prevent mass losses, the carrier gas is returned to the inlet of the pervaporation column by a gas circulation pump.

The products were collected in the condensate collection tubes for 6 hours during an entire con-

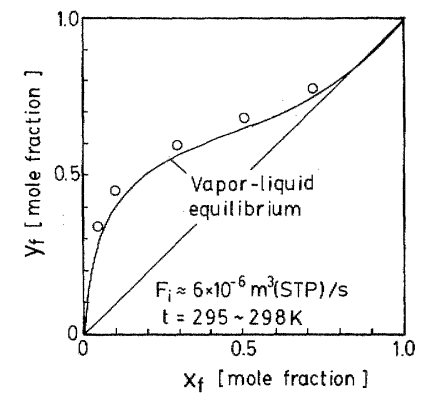

Fig. 2. Sweep gas pervaporation in silicone rubber column

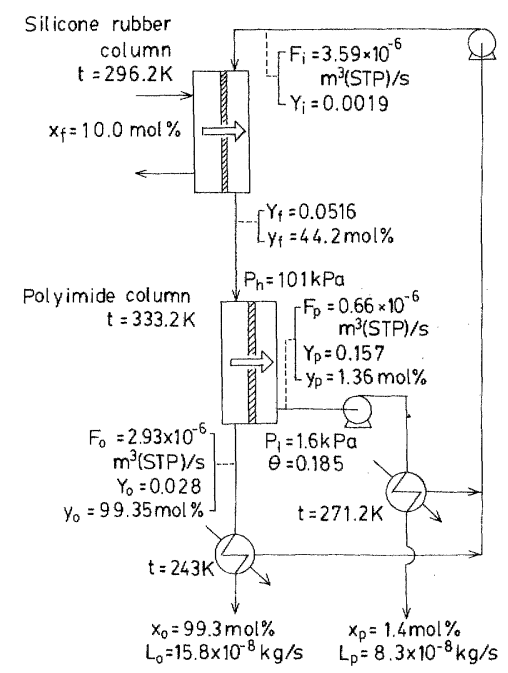

Fig. 3. Example of operating conditions and results of two-stage membrane process

tinuous operation and were analyzed with a refractometer.

\section{Results}

\subsection{Pervaporation performance of the silicone rubber} column

Figure 2 shows the pervaporation performance of the silicone rubber column for ethanol-water mixtures as a relationship between the ethanol concentration of the feeds and that in the permeates. The carrier gas from the silicone rubber column is a little richer in ethanol than the mole fraction of the ethanol-water vapor at vapor-liquid equilibrium at atmospheric pressure. These results are very similar to that reported by Okamoto et al. ${ }^{9)}$ using silicone rubber films.

\subsection{Separation performance of the two-stage mem-} brane process

Figure 3 shows an example of operating conditions and experimental results of the two-stage membrane separation process. An ethanol-water solution of $10 \mathrm{~mol} \%$ ethanol was fed into the silicone rubber column at room temperature. At the outlet of the column the mole fraction of the substrate (ethanol and water) in the carrier gas, $Y$, was $5.16 \%$. This agrees with the calculated value of the saturation 
concentration, $5.2 \%$, in an inert gas at the operating temperature. The ethanol mole fraction of the substrate vapor excluding the carrier gas in the outlet ternary gas, denoted by $y$ in the figure, was $44 \mathrm{~mol} \%$. The ternary gas fiows into the shell side of the polyimide column and $18.5 \%$ of the inlet gas permeated through the membranes; that is, the stage cut, $\theta$, was 0.185 . Water vapor permeates preferentially through the polyimide membrane and the condensate in the permeate side condenser had a $1.4 \mathrm{~mol} \%$ ethanol content. The vapor in the mixed gas in the reject side is enriched by the residual ethanol vapor and the concentration of the condensate in this side was 99.3 $\mathrm{mol} \%$. If the heat-exchange areas of the condensers is sufficiently large and the carrier gas at the condenser outlet is saturated by the substrate vapors, the fraction of vapors in the carrier gas returning to the pervaporation column for circulation is evaluated to be lower than $0.2 \%$.

There was a difficulty in determining the accurate condensate flow rate, $L_{\mathrm{p}}$, at the permeate side condenser because the condensate was nearly $99 \mathrm{~mol} \%$ water and was held up appreciably on the fins in the condenser. For this reason, the value of $L_{\mathrm{p}}$ shown in Fig. 3 is a calculated one, based on the value of $y_{\mathrm{f}}$.

The concentrations of ethanol in the condensed product liquids in the rejection and the permeation streams, $x_{\mathrm{o}}$ and $x_{\mathrm{p}}$, are plotted against the stage cuts, $\theta$, for the polyimide column in Figs. 4(a) (c).

For the experimental runs in Fig. 4(a) and 4(b), the ethanol concentrations of the feed ethanol-water mixture were $10 \mathrm{~mol} \%$ and of $5.5 \mathrm{~mol} \%$ respectively, and the stage cuts, $\theta$, were changed by the carrier gas flow rates while $P_{l}$ was kept constant. For the runs in Fig. 4(c), $P_{l}$ was varied by using different diaphragm vacuum pumps, while the carrier gas flow rate was kept constant. When the stage cut at the polyimide column approaches zero, the composition of the condensate in the rejection side of the column becomes closer to that of the pervaporated vapor through the silicone rubber column. As the stage cut increases, the composition in the rejection side of the column approaches $100 \%$ ethanol. The composition of the rejection stream vapor can easily exceed the azeotropic composition of ethanol-water mixture $(89 \mathrm{~mol} \%)$ because the gas-phase separation process occurs also at this composition. From Figures 4(a)-(c), the compositions of the condensates in the permeate side are lower than $2 \mathrm{~mol} \%$ ethanol.

\section{Discussion}

The data obtained in these experiments for separation of ethanol solutions allow an estimation of the permeation rates of the three components through the polyimide membrane. The assumption was made that the permeation rate of each component

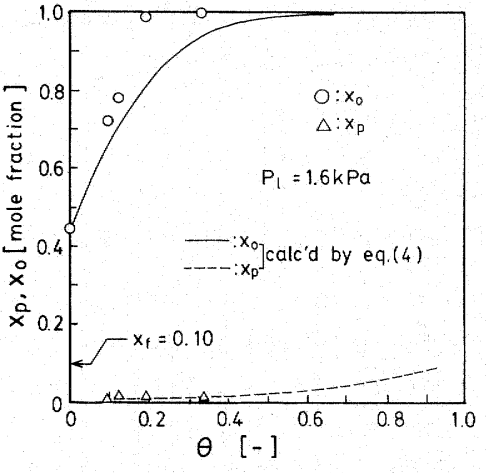

Fig. 4(a). Separation performance of two-stage membrane separation process $\left(x_{f}=10 \mathrm{~mol} \%\right)$

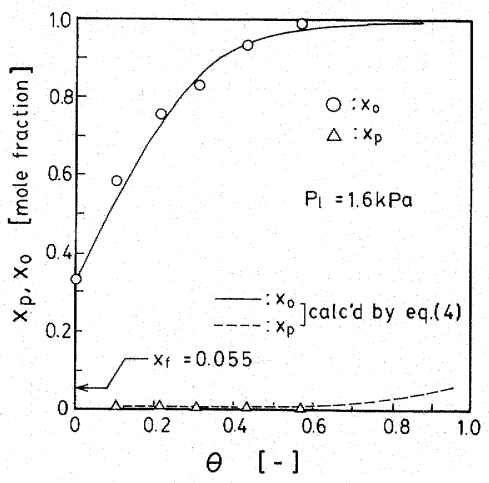

Fig. 4(b). Separation performance of two-stage membrane separation process $\left(x_{f}=5.5 \mathrm{~mol} \%\right)$

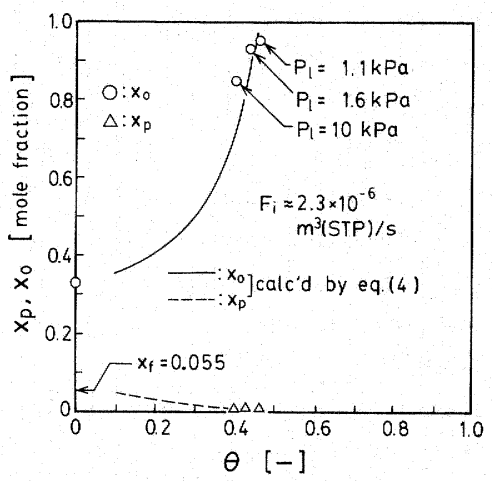

Fig. 4(c). Separation performance of two-stage membrane separation process (effect of pressure at permeate side)

does not affect those of the others. Since the variations of partial pressures of the feed-side flow along the column length are small for helium and ethanol, the fundamental formulas for the permeation of these components are given as follows. ${ }^{2)}$ Helium:

$$
F_{\mathrm{p}}\left(1-Y_{p}\right)=\left(P R_{3}\right) S\left[P_{h}\left(1-Y_{o}\right)-P_{l}\left(1-Y_{p}\right)\right]
$$

Ethanol:

$$
F_{p} Y_{p} y_{p}=\left(P R_{1}\right) S\left(P_{h} Y_{o} y_{o}-P_{l} Y_{p} y_{p}\right)
$$

The partial pressure of water vapor varies along the feed-side flow of the column. Therefore, a differential equation with respect to the flow rate off water vapor is given by Eq. (3), which is solved under the boundary 
conditions obtained from the experimental data at the inlet and the reject outlet points.

$$
\begin{aligned}
& -\frac{d F_{s} Y_{s}\left(1-y_{s}\right)}{d l}= \\
& \quad\left(P R_{2}\right)\left(S / l_{c}\right)\left[P_{h} Y_{s}\left(1-y_{s}\right)-P_{l} Y_{p}\left(1-y_{p}\right)\right]
\end{aligned}
$$

Because the polyimide hollow-fiber membrane used in this study was reported as an asymmetric type (a skin layer and a porous layer exist in the cross section $)^{6)}$ and there is no information about its effective thickness, permeabilities are represented in the form of permeation rates, $P R$, in these equations.

The permeation rates are evaluated from a series of data for the $5.5 \mathrm{~mol} \%$ ethanol solution shown in Fig. 4(b). The permeation rate for each component is plotted against the stage cut in Fig. 5(a). The concentration of the condensate could be determined with an accuracy of $\pm 0.2 \mathrm{~mol} \%$. The error range estimated from the accuracy is also shown in Fig. 5(a) for ethanol. The ideal separation factors for pairs of the components are roughly $\alpha$ (water/ethanol $)=200, \alpha($ helium $/$ ethanol $)=25$, and $\alpha($ water $/$ heli$u m)=8$. These separation factors are similar to that evaluated from the permeation rate data of pure vapors through polyimide hollow fiber reported by Nakamura et $a l^{7,8)}$ The interactions for the permeation rates among the components seem to be small enough for practical applications.

Figure 5(b) shows the mean partial pressures of water vapor in both sides of the polyimide column. A comparison of Figs. 5(a) and (b) reveals that the water permeation rates are not affected by its vapor pressure, but that the dissolved water in the membrane slightly affects the permeation rates of the other components in a higher water-concentration range, or in a low $\theta$ range.

Requirements for the carrier gas in the two stage membrane separation process are that it must have a relatively large permeation rate and must carry the permeated condensable vapors to the condenser through the vacuum pump without condensing the vapor in the line. In Fig. 6, the permeation rates through the polyimide membrane of water vapor and ethanol vapor and compared with those of $\mathrm{He}, \mathrm{N}_{2}$ and $\mathrm{O}_{2}$. These measurements were performed at the condition of $303 \mathrm{kPa}$ in the feed side and atmospheric pressure in the permeate side. This figure suggests strongly that helium, the permeation rate of which falls between those of ethanol and water vapors, is a suitable carrier gas.

Theoretical calculations using the average values of these measured permeation rates can predict the performance of the two-stage membrane separation process under the assumption that the substrate vapor is saturated at the outlet of the silicone rubber column.

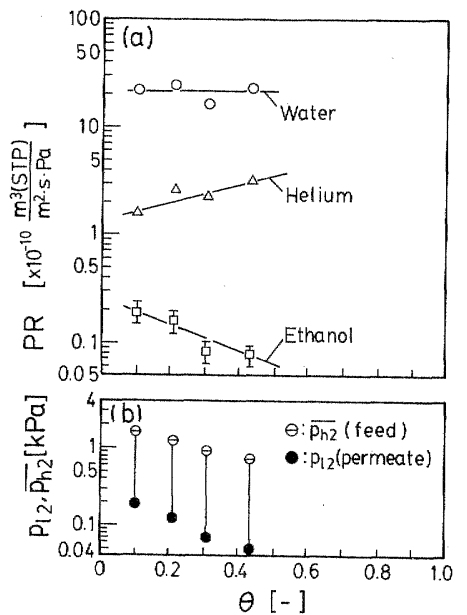

Fig. 5(a). Permeation rate of each component through the polyimide column

Fig. 5(b). Partial pressures of water vapor in both sides of the polyimide column

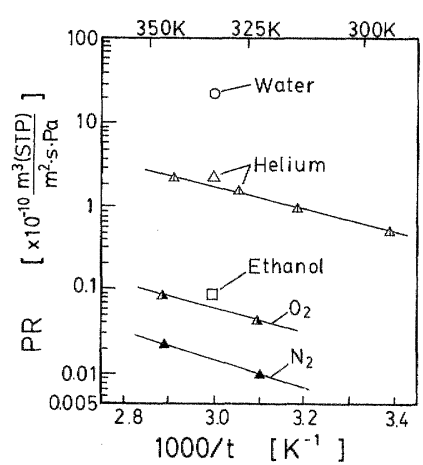

Fig. 6. Permeation rates of inert gases and vapors of water and ethanol through polyimide column

The differential equations with respect to the flow rate of each component as given by Eq. (4) were integrated over the length of the polyimide column ${ }^{2}$.

$$
\begin{aligned}
& -\frac{d F_{s i}}{d l}= \\
& \left(P R_{i}\right)\left(S / l_{c}\right)\left(P_{h} F_{s i} / F_{s}-P_{l} F_{p i} / F_{p}\right) \quad(i=1,2,3)
\end{aligned}
$$

Figures 4(a)-(c) show the values calculated by Eq. (4), where the full lines are the condensate concentration profiles for the reject streams and the dashed lines are those for the permeate streams. The above theoretical predictions confirm that the more the stage cut increases, the more the enrichment of ethanol progresses. There are two ways to change the stage cut. One is by changing the feed flow rate while the pressure in the permeate side is kept constant, and the other one is by changing the pressure in the permeate side. Comparing these theoretical calculations in Fig. 4(b) and 4(c), we can see that high separation performance can be attained at conditions of lower pressure in the permeate side, as was reported for the binary membrane separation. ${ }^{10)}$ 


\section{Conclusion}

A two-stage membrane separation process utilizing two hollow-fiber column, made of silicone rubber and polyimide membranes respectively, was developed. This process provided highly purified product streams at both outlets (the shell side and the permeate side) of the polyimide column. For the polyimide column, the permeation rates of three components were measured and theoretical predictions were compared with experimental results. The high separation performance of the process must be due to the high separation factor of water vapor to ethanol vapor, $\alpha$, of 200 , on the polyimide membrane. The actual degree of separation obtained in the process depends mainly on the fraction of permeate to feed, which is the stage cut, in the polyimide column.

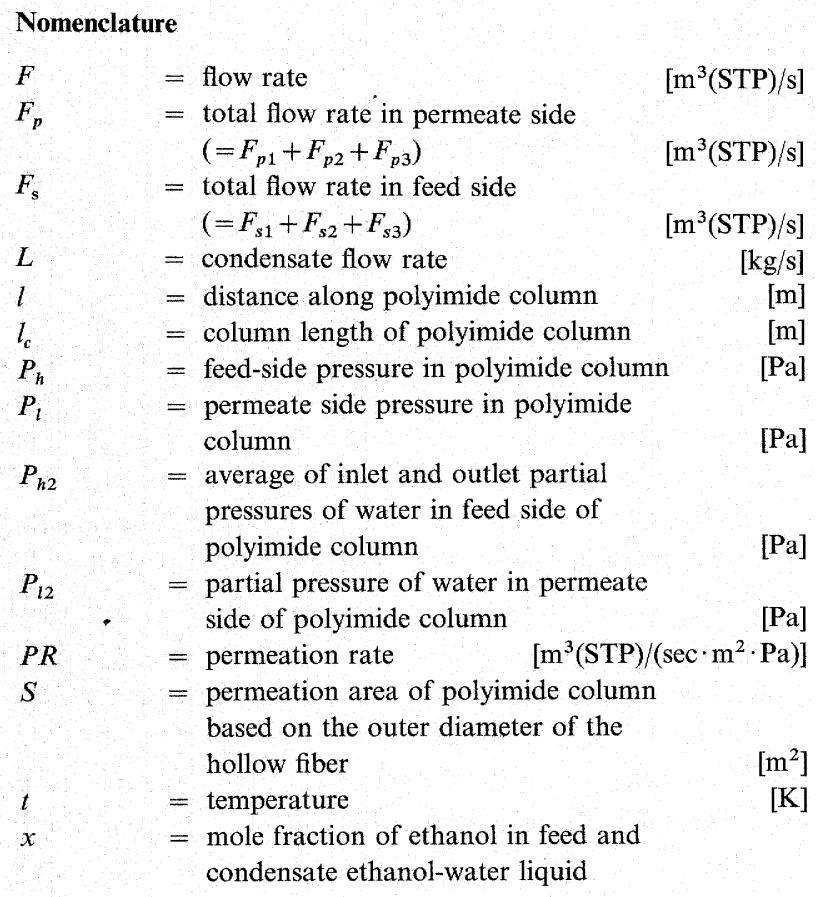

$\begin{array}{lll}Y & \text { mixture } & {[-]} \\ y & = & \text { vapor (ethanol and water) mole fraction } \\ & \text { in carrier gas } \\ = & \text { mole fraction of ethanol in vapor } \\ & \text { contained in carrier gas } \\ \alpha(i / j) \quad & \text { ideal separation factor based on } \\ & \text { permeation rates of pure components } \\ & \left(P R_{i} / P R_{j}\right)\end{array}$

$\langle$ Subscripts〉

$$
\begin{array}{ll}
f & =\text { feed flow to polyimide column } \\
i & =\text { carrier gas feed to silicone rubber column } \\
o & =\text { reject flow } \\
p & =\text { permeate flow } \\
s & =\text { along shell side of polyimide column } \\
1 & =\text { ethanol } \\
2 & =\text { water vapor } \\
3 & =\text { helium }
\end{array}
$$

\section{Literature Cited}

1) Hoover, K. C. and S.-T. Hwang: J. Membrane Sci., 10, 253 (1982).

2) Hwang, S.-T. and K. Kammermeyer: "Membrane in Separations", John Wiley \& Sons, Inc., New York (1975).

3) Kimura, S. and T. Nomura: SEISAN KENKYU, 35, 289 (1983).

4) Kikuchi, M., S. Asano and K. Ninomiya: Kagaku Kōgaku, 53, 51 (1989).

5) Kita, H., S. Sasaki, K. Tanaka, K. Okamoto and M. Yamamoto: Kagaku Kogaku Ronbunshu, 15, 604 (1989).

6) Nakamura, A.: KOBUNSHI, 35, 1081 (1986).

7) Nakamura, A, S. Ono and K. Ninomiya: Kagaku Kogaku, 51, 695 (1987).

8) Nakamura, A and H. Makino: $M A K U(M E M B R A N E), 12$, 287 (1987).

9) Okamoto, K., S. Nishioka, S. Tsuru, S. Sasaki, K. Tanaka and H. Kita: Kobunshi Ronbunshu, 45, 993 (1988).

10) Peinenann, K.-V., J. M. Mohr and R. W. Baker: AIChE Symp. Ser. 250, 82, 19 (1986).

11) Senguputa, A. and K. K. Sirkar: AIChE J., 33, 529 (1987).

K] 12) Seok, D. R., S. G. Kang and S.-T. Hwang: J. Membrane Sci., 33, 71 (1987). 